\title{
Psychometric testing on two weight stigma instruments in Iran: Weight Self-Stigma Questionnaire and Weight Bias Internalized Scale
}

\author{
Chung-Ying Lin ${ }^{1} \cdot$ Vida Imani ${ }^{2} \cdot$ Pauline Cheung ${ }^{1} \cdot$ Amir H. Pakpour ${ }^{3,4} \mathbb{C}^{(}$
}

Received: 10 November 2018 / Accepted: 20 April 2019

(c) Springer Nature Switzerland AG 2019

\begin{abstract}
Purpose This study aimed to comprehensively and thoroughly examine the psychometric properties of two commonly used weight-related self-stigma instruments on Iranian adolescents with overweight or obesity: Weight Self-Stigma Questionnaire [WSSQ] and Weight Bias Internalization Scale [WBIS].

Methods After ensuring the linguistic validity of both the WSSQ and WBIS in their Persian versions, 737 Iranian adolescents with overweight or obesity (male $=354$; mean age $=15.8 \pm 1.3$ years; body mass index $=30.0 \pm 4.8 \mathrm{~kg} / \mathrm{m}^{2}$ ) completed both questionnaires and other relevant measures regarding their depression, anxiety, stress, dietary self-efficacy, weight efficacy lifestyle, quality of life, body fat, self-esteem, body shape preoccupation, and sleepiness.

Results In the scale level, the confirmatory factory analysis verified the two-factor structure for the WSSQ and the singlefactor structure for the WBIS. The factorial structures were further found to be invariant across gender (male vs. female) and across weight status (overweight vs. obesity). Additionally, both the WSSQ and WBIS had promising properties in internal consistency, test-retest reliability, separation reliability, and separation index. In the item level, all items but WBIS item 1 (infit mean square $=1.68$; outfit mean square $=1.60$ ) had satisfactory properties in factor loadings, corrected item-total correlation, test-retest reliability, and infit and outfit mean square. Moreover, all the items did not display substantial differential item functioning (DIF) across gender and across weight status.

Conclusion Both the WSSQ and WBIS were valid instruments to assess the internalization of weight bias for Iranian adolescents with overweight or obesity.

Level of evidence Level V, cross-sectional descriptive study.
\end{abstract}

Keywords Adolescent $\cdot$ Confirmatory factor analysis $\cdot$ Reliability $\cdot$ Rasch $\cdot$ Validity

\section{Introduction}

The impact of overweight and obesity on humans has been widely studied in different aspects, including physiological, social, and psychological health [1-3]. Internalized stigma

Amir H. Pakpour

Pakpour_Amir@yahoo.com; apakpour@qums.ac.ir

1 Department of Rehabilitation Sciences, The Hong Kong Polytechnic University, Hung Hom, Hong Kong

2 Pediatric Health Research Center, Tabriz University of Medical Sciences, Tabriz, Iran

3 Social Determinants of Health Research Center, Qazvin University of Medical Sciences, Shahid Bahonar Blvd, Qazvin 3419759811, Iran

4 Department of Nursing, School of Health and Welfare, Jönköping University, Jönköping, Sweden from weight (or weight-related self-stigma) is suggested to be one of the key factors for the impaired social and psychological health conditions caused by overweight or obesity $[4,5]$. Specifically, weight-related self-stigma is a process of self-devaluation for people who internalize the negative evaluation of their weight regardless of their weight status [5-7]. That is, after an individual believes that a person with overweight is worthless and inferior to people with healthy weight, the individual endorses this negative concepts to himself or herself. Through this process, people who have weight-related self-stigma are suggested to have self-prejudice and self-discrimination reactions $[5,6,8]$. As a result, higher weight-related self-stigma was associated with the following outcomes: being teased and bullied, increased anxiety and depression, and decreased physical activity [5, $7,9]$. Given that the interest in studying social health and 